\title{
Correction to: Baton Rouge as a Living Environment
}

\section{Correction to: \\ Chapter 8 in: O. Kühne and C. Jenal, The Multivillage-Metropolis Baton Rouge, RaumFragen: Stadt - Region - Landschaft, https:// doi.org/10.1007/978-3-658-30716-5_8}

Due to an error during production, the wrong content was accidentally included as video 1 . This has now been corrected. 